\title{
All hands to the pump
}

\author{
Lenny Winkel and colleagues found that strong arm muscles can be required to test South \\ Sumatran groundwater samples for arsenic content.
}

\begin{abstract}
What was the objective of the work?
The idea of a prediction map for arsenic in groundwater was born from an interdisciplinary research team of geochemists and geostatisticians. It is known that areas affected by arsenic contamination, such as Vietnam and Bangladesh, are characterized by flat topography, young geological deposits of the Cenozoic era and fine and medium soil textures, so we thought that arsenic contamination may be predictable from surface parameters. We had already collected a substantial amount of groundwater data from affected areas to calibrate the prediction model. The main goal of our prediction maps was to create a tool to quickly locate potential risk areas for groundwater contamination with arsenic.
\end{abstract}

Why did you choose this location? Once we had put together our prediction map, the east coast of Sumatra appeared as a large high-risk area, comparable in size to the risk area in Bangladesh (about $100,000 \mathrm{~km}^{2}$ ), but no groundwater quality studies had been carried out there. We decided to do our groundwater survey in the lowlands of the province of South Sumatra, a flat region dissected by various rivers and characterized by young sedimentation. as it looks similar to other areas in Southeast Asia where arsenic-rich groundwaters are present. Groundwater is already a common source of drinking water in South Sumatra. Owing to the steadily increasing population resulting from the Indonesian transmigration programme - which moves landless people from densely populated areas (such as Java) to less crowded islands in the Indonesian archipelago - we expect groundwater to become an even more important source of drinking water.



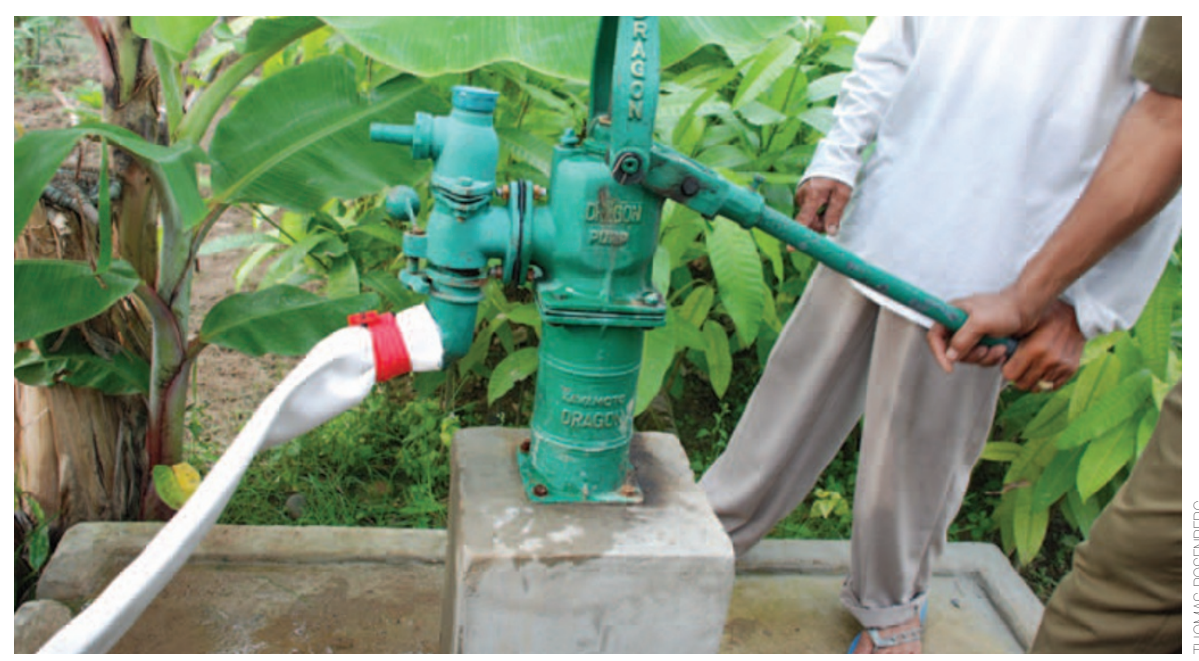

Hand pump for groundwater. Up to 45 minutes of pre-pumping finally yielded reliable samples.

'real' groundwater without changing its chemical composition during pumping or sampling. Before we could take a sample we flushed the tubewells with at least three times the well volume to remove standing water. This required considerable training of arm muscles (for wells equipped with hand pumps) as pre-pumping of 45 minutes was often needed. Then we measured the $\mathrm{pH}$, redox potential, electrical conductivity and dissolved oxygen content, and filled sampling bottles while trying to prevent the water coming into contact with air. Occasionally, we had to tinker with material available on site. For example, when sampling wells from high-positioned tubes, we attached the bucket to the tube with a piece of garden hose and held it up above our heads in order to be as close as possible to the outflow.

\section{Did you encounter any difficulties?}

We had planned to take samples on a regular grid along roads that we recognized on maps and satellite images, at distances of 5-10 km, covering the whole study area. But this proved to be impossible: some of those roads were in such bad condition that we returned with no more than three samples after a day-long bumpy car ride. To bring back at least 100 samples, we had to split up the team, with the driver going back and forth between sampling sites.

What was the highlight of the expedition? The people we met during the fieldwork were incredibly friendly, and happy to help. After determining our target position for sampling by GPS, we went from door to door asking for the nearest tubewell. We talked to families or heads of the village and most of them were happy for us to take a sample of their well. Often we were offered tea and fresh fruit after the sampling.

Any ideas for future research projects? In our data we saw that the recent sedimentation history of the South Sumatra Basin might have been quite different compared with continental Southeast Asia. This means that the three-dimensional geology is even more important in interpreting the arsenic risk maps than we had thought. We now intend to include geological information at depth to see if we can improve the prediction.

This is the Backstory to the work by Lenny Winkel and colleagues, published on page 536 of this issue. 